\title{
Polyetheretherketone Rods in Lumbar Spine Degenerative Disease: Mid-term Results in a Patient Series Involving Radiological and Clinical Assessment
}

\author{
Ahmet OGRENCI ${ }^{1}$, Orkun KOBAN ${ }^{1}$, Onur YAMAN²${ }^{2}$, Mesut YILMAZ ${ }^{1}$, Sedat DALBAYRAK ${ }^{1}$ \\ ${ }^{1}$ Neurospinal Academy, Department of Neurosurgery, Istanbul, Turkey \\ ${ }^{2}$ Koc University, School of Medicine, Department of Neurosurgery, Istanbul, Turkey \\ The study has been presented as an oral presentation at the $12^{\text {th }}$ International Turkish Spine Congress between 19 and 22 April 2017 at Antalya, Turkey
}

Corresponding author: Ahmet OGRENCl drahmetogrenci@gmail.com

\section{ABSTRACT}

AIM: To evaluate the satisfaction of patients operated due to degenerative lumbar spinal diseases with dynamic stabilization placing polyetheretherketone (PEEK) rods and to share their radiological and clinical results (mid-term) with visual analogue scale (VAS) and Oswestry disability index (ODI) scores.

MATERIAL and METHODS: The preoperative and postoperative low back pain, leg pain VAS and ODI scores of 172 patients who were operated for degenerative spinal diseases, were evaluated. Preoperative and postoperative lumbar lordosis were compared. The patients included to the study were evaluated postoperatively around the $2^{\text {nd }}$ year with lumbar MRI by means of adjacent segment disease (ASD) and additional problems.

RESULTS: A statistically but not radiologically-by means of sagittal profile reconstruction-significant increase in lumbar lordosis angle was achieved. Significant improvement was observed in the comparison of preoperative and postoperative period in the analysis of patients' preoperative low back pain $(p<0.0001)$, and decompression-related leg pain VAS scores $(p<0.0001)$. Significant improvement was also observed in the ODI scores of the patients $(p<0.0001)$. Among 172 patients with dynamic stabilization, there were 10 patients who underwent reoperation (5.8\%).

CONCLUSION: Although it is statistically significant, it can be seen that the lumbar lordosis can not be corrected at significant degrees radiographically in the operations performed with the PEEK rod. Dynamic stabilization with PEEK rod is insufficient for sagittal correction, but the mid-term results reached satisfactory reoperation rates clinically outcomes. Rate of ASD is quite low in stabilization with PEEK rod.

KEYWORDS: Adjacent segment disease, Degenerative spinal disease, Dynamic stabilization, Lumbar lordosis, PEEK rod

\section{INTRODUCTION}

S tabilization is sometimes required to relieve the symptoms of degenerative spinal disease. Rigid systems are commonly used to solve these problems. Although the short-term results of these implants have been promising, problems occur at high rates during the long-term follow-up.
Most important among these are adjacent segment disease (ASD) and pseudoarthrosis (3). Rigid stabilization decreases the range of motion of the spinal column and causes increased stress on the adjacent segments postoperatively. Increased stress on the bone-screw interface also leads to instrumentation problems and pseudoarthrosis (35). Dynamic
Ahmet OGRENCI : (1) : 0000-0002-7580-0227 Orkun KOBAN

Onur YAMAN

(D) : 0000-0002-4721-0502

(D) : 0000-0002-2038-1643
Mesut YILMAZ

(1) : 0000-0001-8633-7883
Sedat DALBAYRAK (D) : 0000-0001-9538-5190 
instrumentation has several advantages in this regard because it provides stabilization, balances the stress on the adjacent segments, and decreases the risk of pseudoarthrosis. Almost all degenerative spinal diseases involve segmental instability (14). Our hypothesis is; in majority of degenerative spinal diseases, the use of a dynamic polyetheretherketone (PEEK) rod may solve many problems in the short and long term. Minimal support is sufficient for segmental instability if there is no obvious instability. We share the mid-term results of patients with degenerative spinal disease who have been treated with dynamic stabilization using PEEK rods.

\section{MATERIAL and METHODS}

This was a retrospective clinical and radiological study. Ethical committee approval was obtained from accompanying institutions. A total of 172 patients with degenerative spinal disease (foraminal and spinal stenosis, degenerative listhesis, and degenerative disc disease) were included in the study. All patients had been operated with titanium screws, nuts, and PEEK rods between 2012 and 2015 in three centers by four neurosurgeons. Inclusion criteria in our study were as follows: patients who had undergone lumbar surgery for the first time, those who had degenerative listhesis on dynamic lumbar radiographs ( $>3 \mathrm{~mm}$ translation), those with coronal angulation $<10^{\circ}$, and those who showed iatrogenic instability following a preoperative facet joint resection and who could be followed up after 2 years with magnetic resonance imaging (MRI) and physical examination.

The following parameters were recorded in all patients: preoperative and postoperative low back pain and dominant side leg pain visual analogue scale (VAS) scores, using a 0-10 point VAS; preoperative and postoperative functional outcomes using the Oswestry disability index (ODI) questionnaire (translated into Turkish); and preoperative and postoperative lumbar lordosis using neutral standing X-Rays. Further, we compared patients who underwent short-segment (one spinal motion segment) stabilization with patients who underwent long-segment (more than one spinal motion segment) stabilization. Twenty patients with iatrogenic instability had undergone unilateral facetectomy. These patients were also compared with other patients. With regard to the diagnoses of the patients in the study, some patients had a combination of several spinal disorders, whereas some had only one diagnosis.

Statistical evaluation of the low back pain VAS scores and ODI scores was also performed to distinguish the results of each of the four surgeons in this multicenter study.

\section{Surgical Technique}

The patient is placed in prone position. An appropriate skin incision is performed under general or spinal anesthesia according to the surgical planning. After opening of the fascia and paravertebral muscles, the ligamentous structures around the facet joint, where the uppermost screw is to be placed, are protected, and lateral screwing is performed in this segment as much as possible. Total laminectomy is not performed in any patient. Following decompression (hemilaminectomy, microdiscectomy, or bilateral decompression with unilateral approach), segments with instability due to preoperative scannings are stabilized. Stabilization is performed to increase foraminal height in patients with foraminal stenosis and in patients with iatrogenic instability following facet joint resection. After the additional interventions are completed, the operation table is deflexed with taking into consideration of spinopelvic parameters, and the rod is placed with the patient in extension. The system is then tightened by fastening the nut in the screw head with torque applier using $5 \mathrm{~N}$ pressure. During screwing, care is taken that the disc structures adjacent to the corpus with the screw at the top and the bottom are not degenerated so much. If disc degeneration is excessive (>Pfirrmann grade 3) or protrusion is present, stabilization is extended such that the disc space is maintained between the instrumented segments.

\section{RESULTS}

The age range of the patients in our study was $26-86$ years (mean 55.6 years). There were 48 male and 124 female patients. The mean follow-up period was 62.7 months (range: 26-76 months).

When analyzing the numbers of levels that had undergone instrumented fusion (screwed vertebral bodies), most patients had three levels fused (70 patients; $40.6 \%$ ), followed by 36 patients with two levels $(20.9 \%), 40$ patients with four levels (23.2\%), 14 patients with five levels (8.1\%), and 10 patients with six levels (5.8\%). The average number of vertebral bodies that had been instrumented was 3.32 .

Significant improvement was observed between the mean preoperative and mean postoperative low back pain VAS scores of the patients $(p<0.0001)$ (Table I). Significant improvement was observed between the mean of the preoperative and postoperative leg pain VAS score (dominant side leg pain) $(p<0.0001)$ (Table I). Significant improvement was also found between the mean preoperative and postoperative ODI scores of the patients $(p<0.0001)$ (Table I). The average preoperative lumbar lordosis angle of the patients was $42.5^{\circ}$ whereas the postoperative average lumbar lordosis angle was $44.0^{\circ}$. There was a statistically significant difference between the pre- and postoperative lumbar lordosis angles $(p<0.0001)$ (Table II).

Statistical evaluation of low back pain VAS scores was performed between patients who underwent unilateral facetectomy in the decompression phase without interbody support and those who did not undergo facetectomy. There was no significant difference in the low back pain VAS and ODI scores between these two patient groups (Table III and IV). There were no significant differences in low back pain VAS and ODI scores between the four surgeons in this study (Table III and IV).

The patients were divided into two groups comparing those treated with short-segment and long-segment stabilization; there was no significant differences in VAS and ODI scores between preoperative and postoperative periods among these patients (Table III and IV). 
Ogrenci A. et al: Dynamic Stabilization with PEEK Rods

Table I: The Comparisons of Pain and Disability Scores Between Preoperative and Postoperative Period

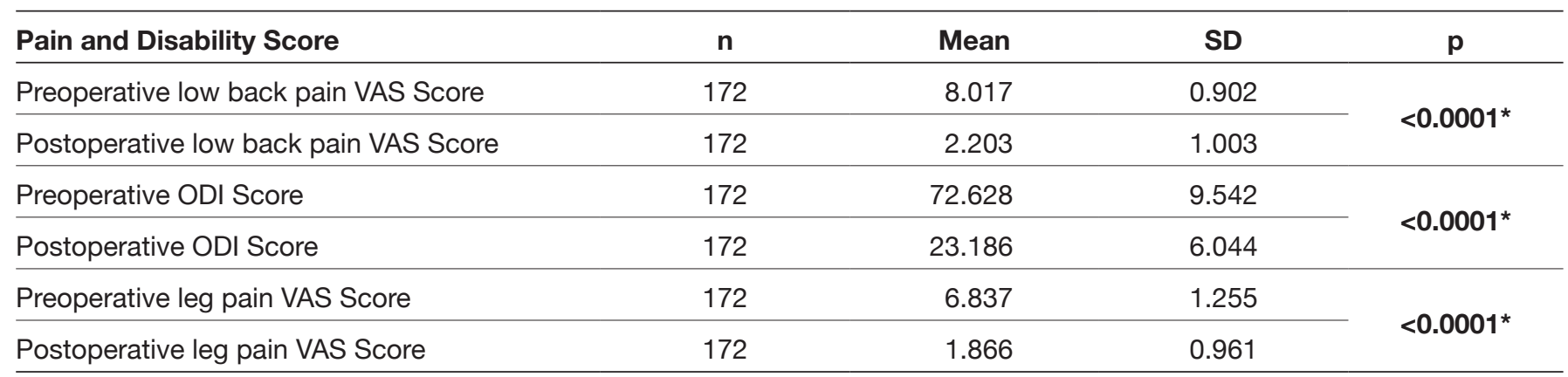

*Wlcoxon Signed Rank test.

Table II: The Comprasion of Lordosis Changes Between Preoperative and Postoperative Periods in Whole Group

\begin{tabular}{lcccc}
\hline Lordosis & $\mathbf{n}$ & Mean & SD & p \\
\hline Preoperative & 172 & 42.517 & 11.204 & $<0.0001^{*}$ \\
\hline Postoperative & 172 & 44.029 & 10.717 & $<$ \\
\hline
\end{tabular}

*Paired sample t test.

Table III: The Comparisons of the Disability Scores Between Preoperative and Postoperative Periods of Patients Who Underwent Different Intervention and Patients Operated by Different Surgeons

\begin{tabular}{|c|c|c|c|c|}
\hline \multirow{2}{*}{ Characteristic } & & \multicolumn{2}{|c|}{ ODI Difference } & \multirow{2}{*}{$\mathbf{p}$} \\
\hline & & Mean & SD & \\
\hline Facetectomy * & No & 10.74 & -49.46 & NS \\
\hline \multirow{2}{*}{ Number of instrumented level * } & Short Segment & 12.28 & -48.89 & \multirow{2}{*}{ NS } \\
\hline & Long Segment & 10.41 & -49.59 & \\
\hline \multirow{3}{*}{ Surgeon ** } & Surgeon 1 & 10.06 & -50.52 & \multirow{3}{*}{ NS } \\
\hline & Surgeon 3 & 6.80 & -46.56 & \\
\hline & Surgeon 4 & 10.87 & -48.52 & \\
\hline
\end{tabular}

*Mann Whitney U test, ${ }^{* * K r u s k a l ~ W a l l i s ~ t e s t . ~}$

Table IV: The Comprasions of Low Back Pain VAS Scores Between Preoperative and Postoperative Periods of Patients Who Underwent Different Intervention and Patients Operated by Different Surgeons

\begin{tabular}{|c|c|c|c|c|}
\hline \multirow{2}{*}{ Characteristic } & & \multicolumn{2}{|c|}{ Low Back pain VAS difference } & \multirow{2}{*}{$\mathbf{p}$} \\
\hline & & Mean & SD & \\
\hline \multirow{2}{*}{ Facetectomy } & No & 1.31 & -5.72 & \multirow{2}{*}{$N^{*}$} \\
\hline & Yes & 1.16 & -6.07 & \\
\hline \multirow{2}{*}{ Number of instrumented level } & Short Segment & 1.31 & -5.90 & \multirow{2}{*}{$\mathrm{NS}^{*}$} \\
\hline & Long Segment & 1.29 & -5.72 & \\
\hline \multirow{3}{*}{ Surgeon } & Surgeon 1 & 1.36 & -5.67 & \multirow{3}{*}{$\mathrm{NS}^{*}$} \\
\hline & Surgeon 3 & 1.03 & -5.75 & \\
\hline & Surgeon 4 & 1.42 & -6.13 & \\
\hline
\end{tabular}

${ }^{*}$ ANOVA. 
The total number of patients who had to be reoperated was $10(5.8 \%)$. In two patients, it was observed that the upperlevel nuts were loosened and displaced posteriorly. In four patients, ASD (two patients having upper and 2 having lower segment disease) was observed. One patient experienced rod breakage. Extruded disc herniation was observed in one patient with ASD at the upper level of instrumentation. Disc degeneration and foraminal stenosis in the adjacent segment at the lower level was found in the remaining three patients.

In four patients, the instrumentation system was extended. In two patients, instrumentation systems were extended due to loosening of the lowest level screw (from L5 to S1). In one patient, the screw was loosened and had dislocated posteriorly; therefore, the system had to be revised and extended. The instrumentation system was further revised in one patient after the diagnosis of sacroiliac joint pain by sacroiliac injection due to screw penetration. All patients benefitted from additional surgery.

None of the patients had instrumentation-related infections. Three patients were treated with antibiotics for superficial wound infection.

\section{DISCUSSION}

Spine degenerative disease begins with the deterioration of disc contents. Subsequently, the load on the facet joints increases, initiating the process of an increasingly unbalanced load distribution on the spine; the load distribution shifts from anterior to posterior $(19,24,33,34)$. Instability between segments may occur as a consequence (14). This leads to accelerated degenerative progression and symptoms, especially axial chronic back pain (36) (Figure 1A-C). As part of the accelerated degenerative process, radicular symptoms increase.

In order to treat segmental instability, fusion surgery has been performed for the last four decades using rigid systems. Patients are satisfied with this surgical treatment because the instrumentation with rigid systems provides stabilization and decompression. However, stabilization with rigid systems limits physiological movements of the spine, increases the stresses at adjacent levels and further system problems due to the high resistance at the bone-screw interface $(4,28)$.

In fact, Turner at al. have developed the terms "better load sharing" and "less bone-screw interface stresses." Systems that cannot share the load properly increase stress on both neighboring tissues and themselves. This leads to problems, such as increasing the risk of avulsion or fracture of the systems at fused level (35).

Dynamic systems provide stabilization and decrease both the load on the adjacent segments and the stresses at the bonescrew interface. In studies of stabilization with rigid systems, it has been reported that the probability of ASD is very high during a 5-10-year follow-up $(3,9,32)$.

Screw revision rates are also increasing over time. One study reported a reoperation rate of nearly $40 \%$, and most of these reoperations $(65 \%)$ were related to the system and the results it produces (21).

Pseudoarthrosis, screw and rod fractures are other important problems. In fact, in many degenerative spinal diseases (spinal stenosis, DDD, listhesis, ASD, recurrent disc herniation etc.) the main problem is not evident instability. Unbalanced segmental load distribution and microinstability are the critical
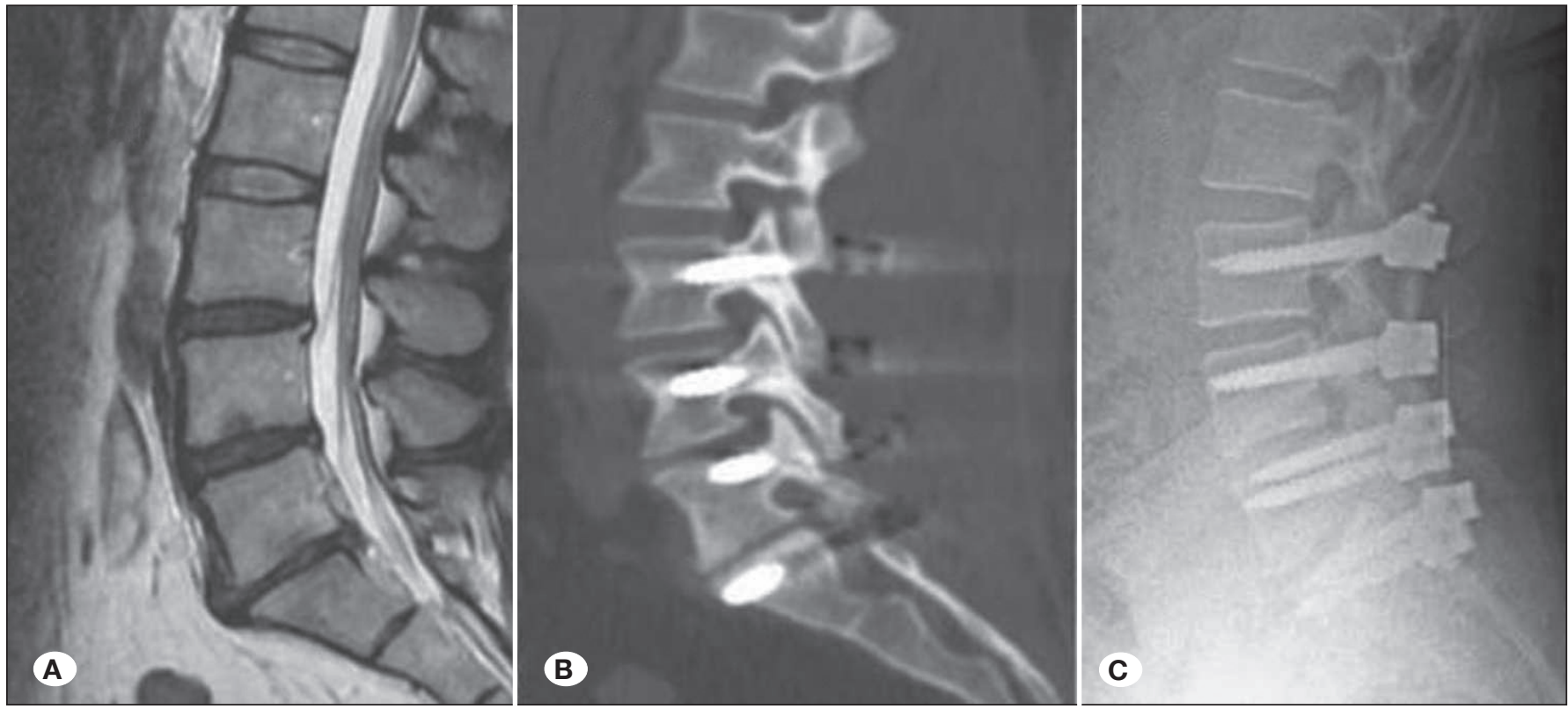

Figure 1: A 44-year-old female patient with chronic low back pain (VAS score: 9) and intermittent left leg pain was examined with MRI and degenerations at L3-4, L4-5 and L5-S1 intervertebral discs were observed (A). Patient was diagnosed as degenerative disc disease and L3-S1 dynamic instrumentation with PEEK rod were performed in patients who did not benefit from conservative methods (B). A postoperative $4^{\text {th }}$ year X-ray examination showed no system problems (C). Postoperative low back pain VAS score was: 2. 
factors in these diseases. To counteract this instability, it is enough to provide minimal support. Stabilization with rigid systems to solve low back pain due to axial loading is a very invasive surgery for these patients. Over the last two decades, dynamic instrumentation has therefore been performed more frequently in this situation, with further increasing usage rates in recent years.

Dynamic systems have been developed based on a rod and screws, but there is still no optimal system. More recently, PEEK rods have been increasingly used. PEEK rods were introduced in the market in 2006. Laboratory studies have shown that they present one of the most suitable systems to maintain spine physiology $(16,28)$.

Previous large series of PEEK rod use have not reported on the long- and mid-term results. For smaller patient groups, presented outcomes usually refer to an approximate 2-year follow-up $(5,12,13,15,18,26)$. To the best of our knowledge, the largest patient series to date has been published by Athanasakopoulos et al., including 52 patients (5). Our study therefore presents the largest number of patients followed up for the longest period of time so far, and was performed as a multicenter study.

Highsmith et al. was the first to report positive results in only three patients (17). After that, biomechanical studies were carried out on cadavers and using the finite element model with the PEEK rod, which was compared to rigid systems and measured patient satisfaction $(16,22,28,30,37)$. In these studies, PEEK rods were determined to have several advantages with regard to load sharing compared with rigid systems. Laboratory tests have shown that the axial load is transmitted to the surrounding tissues, facets, and discs in $33 \%$ of specimens using PEEK rods and in $71 \%$ using titanium rods (2). In fact, improved load sharing is the most important advantage of PEEK rods. In addition to their superior load distribution, they have been shown to provide similarly, or even more, durable stabilization compared to other systems (2). PEEK rods have been shown to reduce the risk of fracture and screw scrapings in the instrumentation system. It has been demonstrated that intradiscal pressure is lower with the PEEK rod than with titanium rods, which reflects the better load sharing. In addition, there are laboratory studies that show that PEEK rods provide adequate stabilization and reduce the range of motion similarly to rigid systems $(1,8,16)$.

The creation of a posterior tension band without attempting to create an arthrosis is actually the "healthiest" aspect of the system, and perhaps it may be the best definition for PEEK rod. This limited support is often sufficient for patients who have degenerative instability.

There are also biomechanical studies of the stability of PEEK rods. Static compressive bending ( $67^{\circ}$ displacement) and torsion testing $\left(30^{\circ}\right.$ rotation) did not break the rod and did not cause plastic deformation (30). Chou et al. stated that there was no loss of stability in PEEK rods, whereas there was loss of stability in titanium rods in a fatigue test involving 90.000 flexion/extension cycles (11). Matthew et al. also argued that Dynesys, PEEK, and titanium structures exhibited similar properties in some directions (22). In a study by Wang et al., the PEEK rod was reported to achieve more favorable results than bone union grafts while producing less stiffness and lower resistance in a canine model (36). Nevertheless, there are also cases of broken PEEK rods being reported (31). We experienced a rod breakage in our study in one patient who had to undergo revision surgery. However, in general, there was no deformation of the original shape or correction between the early postoperative radiographs and the last postoperative radiographs in our patients.

In addition, we observed loosening and posterior migration of nuts in two of our patients that has not been reported in literature to date (Figure $2 \mathrm{~A}-\mathrm{C}$ ). These two patients were reoperated only to have the nuts reinserted and tightened. In
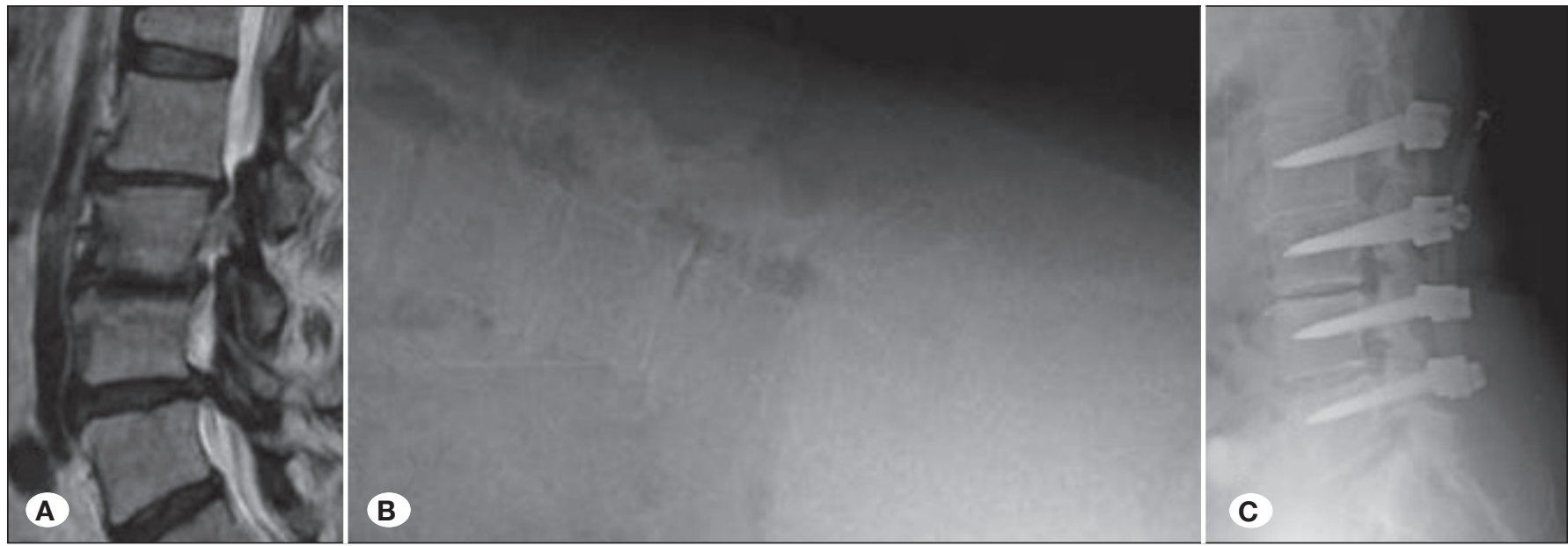

Figure 2: A 58-year-old female patient was admitted with chronic back pain and bilateral leg pain. L2-L3, L3-4, L4-5 spinal stenosis, disc herniations and L3-4 degenerative listhesis were observed in lumbar MRI (A) and hyperflexion lumbar X-rays (B). L2-5 dynamic instrumentation with PEEK rod and decompression to the appropriate levels were performed in the surgical treatment of the patient. In postoperative $2^{\text {nd }}$ month control, nut loosened and rod displacement were observed in the uppermost segment (C). The patient was operated again in order to squeeze the nut. There was no problem to date (nearly 4 years) after the second operation. 
fact, all the nuts were loose in these patients. The problem was thought to be related to a deterioration of the torque setting of the nut driver in the instrumentation set used in the first operation. It was confirmed radiologically that the rod did not loose its elasticity or curve. In addition, in other cases where we performed a revision, we have not seen anomalies other than minor deformations in the places where the nuts rest on the rods. Also, the most common deformations were found to be permanent indentations as described in the article published by Kurtz et al. (20).

When we compare the studies reporting the revision rates of PEEK rod case series, we've found different ratios. Although the series did not comprise a large number of patients, Ormond et al. stated that 8 of 42 patients had to be reoperated; of these and 5 were because of adjacent segment degeneration (23). Athanasakopoulos et al. stated that they did not observe ASD in their group of 52 patients (5). If we consider the longer follow-up period, the ASD rate in our series is extremely low compared with those reported in literature. Only four patients had ASD and had to be reoperated. We believe that the low $A S D$ ratio in our patients is related to our technique that aims to preserve as much of the osseoligamentous structures around the facet joint where the upper and lower screws are placed as possible and not to disrupt the facet joint by placing the screws laterally. Previous studies have used X-rays and disc heights to assess ASD in their patients $(6,12)$. According to Pfirrmann's and Puertas' classification of disc degeneration, such degeneration is possible without a decrease in disc height $(27,29)$. Accordingly, we think that disc height measurement is not sufficient to assess disc degeneration and the presence of ASD. We consider that we have more favorable data for ASD because we included patients who underwent an MRI investigation after the second postoperative year (Figure 3AC).
To the best of our knowledge, there is no other study considering lumbar lordosis with PEEK rods pre- and postoperatively except for Qi et al.'s study that had a limited number of patients and only short-segment stabilized patients (30)

Chen et al. also stated that there was no significant difference in lumbar lordosis between the pre- and postoperative period in other dynamic systems, such as Dynesys (10). Although patients were brought into extension by table deflexion at the end of surgery, the resulting lordosis on postoperative standing X-rays was nearly the same as the preoperative value. In our statistical analysis, a significant difference was found between preoperative lordosis and postoperative lordosis in all patient but results were insufficient. In this regard, we can say that PEEK rods are insufficient for sagittal reconstruction and for increasing lumbar lordosis. Our main goal in this patients group was not to correct lumbar lordosis. In fact, osteotomy was not performed to correct the lumbar lordosis; this can be achieved by titanium rods used in rigid stabilization. We tried to achieve to increase lordosis by deflecting the operation table and using the rod's inherent lordotic angle. But we believe that a few degrees of increase in the postoperative period are radiologically insignificant. The reason for the statistical significance difference between preoperative and postoperative lordosis is that the standard deviation decreases due to the large number of patients in our study.

In one revision case, where the upper screw had become loosened and scraped, the PEEK rod may have caused the loosening because of an insufficient lordosis angle in the sagittal-imbalanced patient.Instability is an expected condition in patients who have undergone facetectomy. There are no generally applicable indications for instrumentation
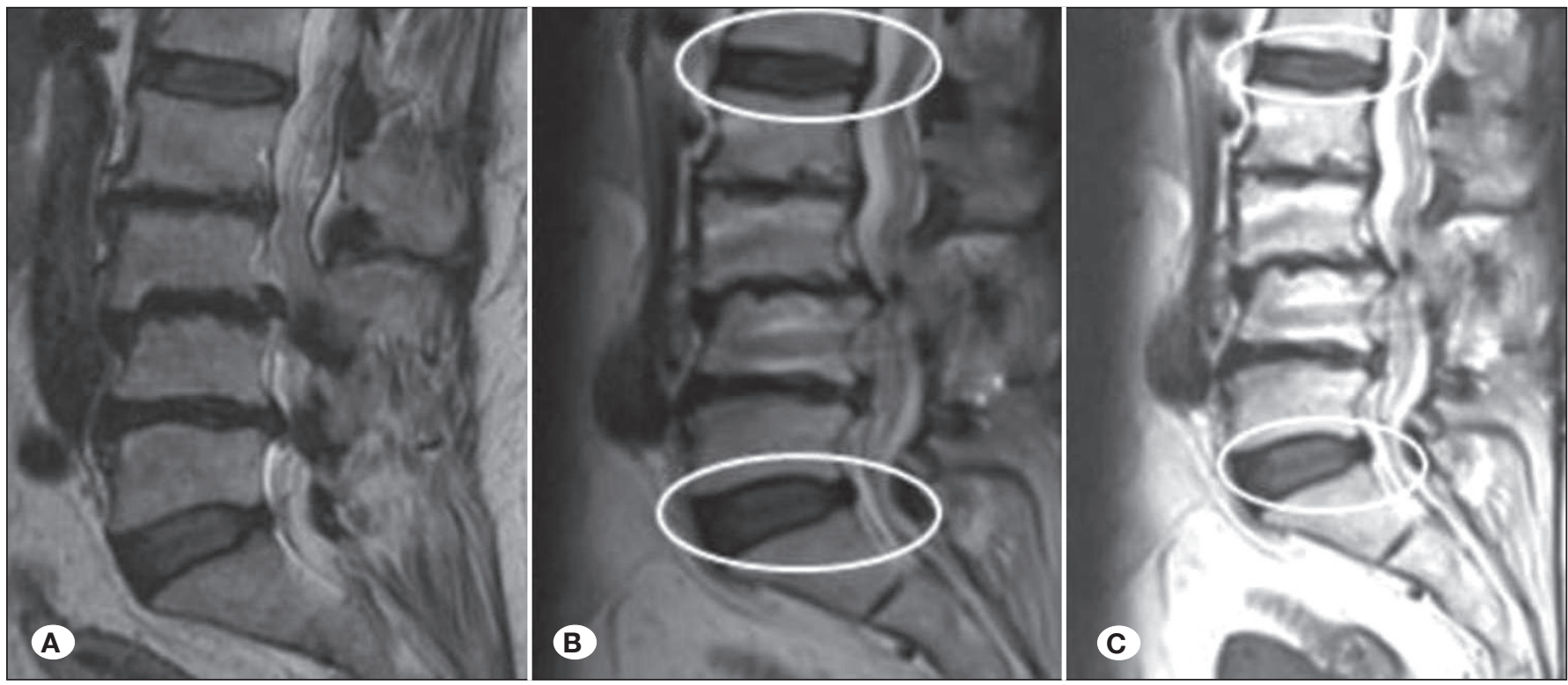

Figure 3: A 62-year-old female patient underwent decompressions and L2-5 dynamic instrumentation wih PEEK rod due to multilevel spinal stenosis, disc herniations, and degenerative listhesis (L3-4). No degeneration was observed in the postoperative $2^{\text {nd }}$ and $4^{\text {th }}$ year evaluations of the patient at both upper and lower adjacent segments (C). 
after facetectomy, but stabilization is usually performed within the currently accepted surgical concept. Rigid stabilizations are frequently performed after facetectomy, but iatrogenic instability after facetectomy can in fact be effectively prevented with dynamic stabilization. Based on their study of the biomechanics of dynamic systems after facetectomy in cadavers, Parikh did not recommend dynamic systems in facetectomy (25). Different from that study, Bruner et al. compared post-facetectomy specimens treated with PEEK rods and others systems, and found all of them to have similar rates of stability (7). There is no study yet which compares pain scores and ODI scores between patients who underwent and those who did not undergo facetectomy in PEEK rod stabilization. In our case series, 20 patients underwent unilateral facetectomy, and no difference in clinical outcomes was observed in their follow-up compared to patients without facetectomy. It is therefore concluded that the PEEK rod can be used safely in patients who have had unilateral facetectomy. There was no statistically significant difference between the four surgeons in terms of low back pain VAS and ODI scores, indicating a low variability due to individual differences in surgical skill and approach. This indicates that the system is effective in achieving pain control independent of the surgeon.

Short-segment stabilizations are known to maintain a more physiological stress loading on the spine than stabilization over long segments (more than one spinal motion instrumentation). Even with rigid systems, short-segment stabilization is thought to not significantly distort spine biomechanics. Therefore, we postulate that short-segment dynamic stabilizations are very close to spine physiology. In literature, there is no clinical evaluation of the differences between short-segment and long-segment stabilization with PEEK rods. In our analysis, there was no difference in the clinical evaluation (VAS and ODI) of patients with short-segment and those with longsegment stabilization with PEEK rods. This finding is much more relevant in long-segment stabilization because it offers an advantage over rigid stabilization, where problems increase and clinical outcomes become less favorable as the number of levels of instrumentation increases.

There are limitations of our study. The use of PEEK rods currently spans a period of 10 years. This study shares midterm results but has no long-term results to report. The cost of the system may appear as a disadvantage at first, but may become advantageous when considering total cost because of shorter operation times, simplicity of application, no requirement for bone graft for fusion, and a potentially shorter hospital stay. Further investigations in larger patient cohorts with different diagnoses and different surgical techniques and treatments are warranted. Patients treated with rigid systems should also be included for comparison.

\section{CONCLUSION}

This study was to date with the largest population and the longest follow-up period examining the clinical and radiological results of patients treated with PEEK rods. Stabilization with PEEK rods is a safe treatment for degenerative spinal diseases based on our mid-term results. In patients who undergo facetectomy, the PEEK rod can also be used safely. Although we found a statistically significant difference between pre- and postoperative lumbar lordosis angles, these were insufficient by means of sagittal profile reconstruction. The revision and $A S D$ rates were found to be low in our study group.

\section{REFERENCES}

1. Abode-lyamah KO, Cox EM, Rajinder Kumar MD, Hitchon PW: $P E E K$ rods decrease intradiscal pressure in levels adjacent to spinal instrumentation. Spine 27(22): 2431-2434, 2002

2. Ahn YH, Chen WM, Lee KY, Park KW, Lee SJ: Comparison of the load-sharing characteristics between pedicle-based dynamic and rigid rod devices. Biomedical Materials 3(4): 044101, 2008

3. Anandjiwala J, Seo JY, Ha KY, Oh IS, Shin DC: Adjacent segment degeneration after instrumented posterolateral lumbar fusion: A prospective cohort study with a minimum five-year follow-up. Eur Spine J 20(11):1951-1960, 2011

4. Asher MA, Carson WL, Hardacker JW, Lark RG, Lai SM: The effect of arthrodesis, implant stiffness, and time on the canine lumbar spine. Clinical Spine Surgery 20(8):549-559, 2007

5. Athanasakopoulos M, Mavrogenis A, Triantafyllopoulos G, Koufos S, Pneumaticos S: Posterior spinal fusion using pedicle screws. Orthopedics 36: 951-957, 2013

6. Benezech J, Garlenq B, Larroque G: Flexible stabilisation of the degenerative lumbar spine using PEEK rods. Advances in Orthopedics 2016:7369409, 2016

7. Bruner HJ, Guan Y, Yoganandan N, Pintar FA, Maiman DJ, Slivka MA: Biomechanics of polyaryletherketone rod composites and titanium rods for posterior lumbosacral instrumentation. Journal of Neurosurgery Spine 13(6):766772,2010

8. Chang TK, Huang CH, Liu YC, Chen WC, McClean CJ, Lai YS, Cheng CK: Biomechanical evaluation and comparison of polyetheretherketone rod system to traditional titanium rod fixation on adjacent levels. Formosan Journal of Musculoskeletal Disorders 4(2):42-47, 2013

9. Cheh G, Bridwell KH, Lenke LG, Buchowski JM, Daubs MD, Kim Y, Baldus C: Adjacent segment disease followinglumbar/ thoracolumbar fusion with pedicle screw instrumentation: $A$ minimum 5-year follow-up. Spine 32(20): 2253-2257, 2007

10. Chen H, Charles YP, Bogorin I, Steib JP: Influence of 2 different dynamic stabilization systems on sagittal spinopelvic alignment. Clinical Spine Surgery 24(1):37-43, 2011

11. Chou WK, Chien A, Wang JL: Biomechanical analysis between PEEK and titanium screw-rods spinal construct subjected to fatigue loading. Clinical Spine Surgery 28(3):121-125, 2015

12. Colangeli S, Barbanti BG, Gasbarrini A, Bandiera S, Mesfin A, Griffoni C, Boriani S: Polyetheretherketone (PEEK) rods: Short-term results in lumbar spine degenerative disease. $J$ Neurosurg Sci 59(2):91-96, 2015

13. De lure F, Bosco G, Cappuccio M, Paderni S, Amendola L: Posterior lumbar fusion by PEEK rods in degenerative spine: Preliminary report on 30 cases. European Spine Journal 21(1): 50-54, 2012

14. Frymoyer JW, Selby DK: Segmental instability: Rationale for treatment. Spine 10(3): 280-286, 1985 
15. Galler RM: The use of PEEK rods in the stabilization of the lumbar spine. In Proceedings of the 79th AANS Annual Scientific Meeting, Eposter, Denver, Colo, USA, 2011

16. Gornet MF, Chan FW, Coleman JC, Murrell B, Nockels RP, Taylor BA, Lanman TH, Ochoa JA: Biomechanical assessment of a PEEK rod system for semi-rigid fixation of lumbar fusion constructs. Journal of Biomechanical Engineering 133(8): 081009, 2011

17. Highsmith JM, Tumialan LM, Rodts Jr GE: Flexible rods and the case for dynamic stabilization. Neurosurgical Focus 22(1): $1-5,2007$

18. Huang W, Chang Z, Song R, Zhou K, Yu X: Non-fusion procedure using PEEK rod systems for lumbar degenerative diseases: Clinical experience with a 2-year follow-up. BMC Musculoskeletal Disorders 17(1):53, 2016

19. Kirkaldy-Willis WH, Farfan HF: Instability of the lumbar spine. Clinical Orthopaedics and Related Research 165:110-123, 1982

20. Kurtz SM, Lanman TH, Higgs G, MacDonald DW, Berven SH, Isaza JE, Phillips E, Steinbeck MJ: Retrieval analysis of PEEK rods for posterior fusion and motion preservation. Eur Spine $J$ 22(12):2752-2759, 2013

21. Martin BI, Mirza SK, Comstock BA, Gray DT, Kreuter W, Deyo RA: Are lumbar spine reoperation rates falling with greater use of fusion surgery and new surgical technology?. Spine 32(19): 2119-2126, 2007

22. Matthew SY, Daniel JC, Boyle CC: In vitro comparison of dynesys, PEEK, and titanium constructs in the lumbar spine. Advances in Orthopedics 2015:895931, 2015

23. Ormond DR, Albert Jr L, Das K: Polyetheretherketone (PEEK) rods in lumbar spine degenerative disease: A case series. Clinical Spine Surgery 29(7):371-375, 2016

24. Ozer AF: Dynamic stabilization of the spine: A new classification system. Turk Neurosurg 20(2): 205-215, 2010

25. Parikh R: Biomechanical comparison of various posterior dynamic stabilization systems for different grades of facetectomy and decompression surgery. Electronic thesis or dissertation. University of Toledo, 2010

26. Pasciak M, Grzywocz J, Nobis A, Pala A, Wadek T: PEEK plastic rods versus titanium 'stiff rods in transpedicular lumbar stabilization. Clinical comparison of short term results. European Spine Journal 18(4): 471-535, 2009
27. Pfirrmann CW, Metzdorf A, Zanetti M, Hodler J, Boos N: Magnetic resonance classification of lumbar intervertebral disc degeneration. Spine 26(17): 1873-1878, 2001

28. Ponnappan RK, Serhan H, Zarda B, Patel R, Albert T, Vaccaro AR: Biomechanical evaluation and comparison of polyetheretherketone rod system to traditional titanium rod fixation. Spine Journal 9(3):263-267, 2009

29. Puertas EB, Yamashita H, Oliveira VMD, Souza PSD: Classification of intervertebral disc degeneration by magnetic resonance. Acta Ortopédica Brasileira 17(1):46-49, 2009

30. Qi L, Li M, Zhang S, Xue J, Si H: Comparative effectiveness of PEEK rods versus titanium alloy rods in lumbar fusion: $A$ preliminary report. Acta Neurochirurgica 155(7):1187-1193, 2013

31. Sarbello JF, Lipman AJ, Hong J, Lawrence J, Bessey JT, Ponnappan RK, Vaccaro AR: Patient perception of outcomes following failed spinal instrumentation with polyetheretherketone rods and titanium rods. Spine 35(17): 843-848, 2010

32. Sears WR, Sergides IG, Kazemi N, Smith M, White GJ, Osburg $B$ : Incidence and prevalence of surgery at segments adjacent to a previous posterior lumbar arthrodesis. Spine Journal 11(1): 11-20, 2011

33. Sengupta DK: Dynamic stabilization devices in the treatment of low back pain. Orthopedic Clinics 35(1): 43-56, 2004

34. Schwab FJ, Nazarian DG, Mahmud F, Michelsen CB: Effects of spinal instrumentation on fusion of the lumbosacral spine. Spine 20(18): 2023-2028, 1995

35. Turner JL, Paller DJ, Murrell CB: The mechanical effect of commercially pure titanium and polyetheretherketone rods on spinal implants at the operative and adjacent levels. Spine 35(21):1076-1082, 2010

36. Wang $\mathrm{N}$, Xie H, Xi C, Zhang H, Yan J: A study to compare the efficacy of polyether ether ketone rod device with titanium devices in posterior spinal fusion in a canine model. Journal of Orthopaedic Surgery and Research 12(1):40, 2017

37. Zhou R, Huang Z, Liu X, Tong J, Ji W, Liu S, Zhu Q: Kinematics and load-sharing of an anterior thoracolumbar spinal reconstruction construct with PEEK rods: An in vitro biomechanical study. Clinical Biomechanics 40:1-7, 2016 